\title{
Mottling Assessment of Solid Printed Areas and Its Correlation to Perceived Uniformity
}

\author{
Albert Sadovnikov, Petja Salmela, Lasse Lensu, \\ Joni-Kristian Kamarainen, and Heikki Kälviäinen \\ Laboratory of Information Processing, \\ Department of Information Technology, Lappeenranta University of Technology, \\ P.O.Box 20, 53851 Lappeenranta, Finland \\ \{sadovnik, psalmela, ltl, jkamarai, kalviai\}@lut.fi
}

\begin{abstract}
Mottling is one of the most important printing defects in modern offset printing using coated papers. Mottling can be defined as undesired unevenness in perceived print density. In our research, we have implemented three methods to evaluate print mottle: the standard method, the cluster-based method, and the bandpass method. Our goal was to study the methods presented in literature, and modify them by taking relevant characteristics of the human visual system into account. For comparisons, we used a test set of 20 grey mottle samples which were assessed by both humans and the modified methods. The results show that when assessing low-contrast unevenness of print, humans have diverse opinions about quality, and none of the methods accurately capture the characteristics of human vision.
\end{abstract}

\section{Introduction}

Printability of paper and print quality are very important attributes when modern printing applications are considered. Especially in prints containing images, high print quality is a basic requirement. Because of non-ideal interactions of paper and ink in high-speed printing processes, there are several undesired effects in prints. One of these effects is mottling which is related to density and gloss of print. It is the uneven appearance of solid printed areas, and it depends on the printing ink, paper type, and printing process. Depending on the phenomenon causing this unevenness, there exists three types of mottling: back-trap mottle (uneven ink absorption in the paper), water-interface mottle (insufficient and uneven water absorption of the paper causing uneven ink absorption), and ink-trap mottle (wet or dry; incorrect trapping of the ink because of tack) [1].

Mottling can be defined as undesired unevenness in perceived print density. In the ISO/IEC 13660 standard, a more technical definition is given [2]: "aperiodic fluctuations of density at a spatial frequency less than 0.4 cycles per millimeter in all directions". In most situations, mottling is a stochastic phenomenon, but different types of noise in print related to mottling can include some form of 
regularity. For example, possibly regular drift in the printing process causes macro-scale noise in print, whereas structures in the paper formation are random in nature and cause micro-scale noise invisible to a human being as such.

Several methods to evaluate mottling by an automatic machine vision system have been proposed. The ISO 13660 standard includes a method for monochrome images. It is based on calculating the standard deviation of small tiles within sufficiently large area 2. In the standard, the size of the tiles is set to a fixed value, which is a known limitation 3 . The first improvement to the standard method was to use tiles of variable sizes 4 . Other methods relying on clustering, statistics, and wavelets have also been proposed [5,6, 7. Other approaches to evaluate greyscale mottling have their basis in frequency-domain filtering 8, and frequency analysis 9. All of the before-mentioned methods are designed for binary or greyscale images. If colour prints were assessed, the correlation of the methods to human assessments would be severely limited. Also the grounds for the methods do not arise from any models for the phenomena causing mottling, nor vision science.

Mottling can be physically defined, but it becomes problematic when a print is perceived. If a person looking at a solid print perceives unevenness, mottling is a problem. Thus, the properties and limits of the human visual system must be taken into account when proper methods to assess mottling are designed. This is especially very important in the assessment of colour images. When perception of image noise is of concern, visual sensitivity to contrast and spatial frequencies of the human visual system (HVS) is independent of luminance within common luminance levels [10. However, contrast sensitivity depends on spatial frequency [11, thus, mottles of different sizes are perceived differently. The peak sensitivity of the HVS is approximately at 3 cycles/degree, and the maximum detected frequency is from 40 cycles/degree (sinusoidal gratings) 12 to over 100 cycles/degree (single cycle) 13 .

The purpose of this work was to compare the artificial methods to a human assessment of mottling samples. In our study, we sought proper background for the methodological selections based on vision science. We implemented three methods based on literature, and modified them as needed to accommodate appropriate knowledge concerning the HVS.

\section{Methods}

We implemented three methods to automatically assess print mottle: the standard method to evaluate image quality of printer systems [2], a cluster method [4, and a band-pass method 86. We slightly modified them as needed to accommodate an appropriate contrast-sensitivity function for the human visual system. To study the correlation of the implemented methods with human perception, we carried out an initial human assessment of 20 mottling samples. We asked experts and laymen to evaluate perceived mottling in the samples. The mean of these subjective assessments was used as a reference into which the results of the methods were compared. 


\subsection{Standard Method}

The ISO 13660 standard is designed for assessing print quality of office equipment that produce monochrome output [2]. The density attributes for large print areas (larger than $21.2 \mathrm{~mm}$ squared) include graininess and mottling. In the standard, a fixed value has been chosen to separate this two forms of print unevenness. Aperiodic fluctuations of print density at spatial frequencies higher than 0.4 cycles/degree are considered as graininess, whereas frequencies lower than the limit are mottling. The standard method is presented in Algorithm 1.

\section{Algorithm 1. Standard method}

1: Divide the region of interest into tiles.

2: Compute the density means within each tile.

3: Compute the standard deviation of the means as the measure of mottling.

In Step 1, the region of interest is divided into tiles of size $1.27 \mathrm{~mm}$ squared. Within each tile, 900 independent measurements of density are made.

\section{$2.2 \quad$ Cluster Method}

This method is based on the idea by Wolin [4. In this method, the raster image is filtered with a low-pass filter, and thresholded separately on both the lighter and darker sides of the median grey value. Geometric measures of the thresholded blobs (mottles) are used as features. In our implementation each blob is weighted by its size and contrast, and the weighted number of blobs is used as the mottling index of the sample. The method is shown in Algorithm 2 .
Algorithm 2. Cluster method
1: Blur the image.
2: Threshold the image on both sides of the image median.
3: Remove small blobs and break connections between the blobs.
4: Remove partially cropped blobs near the image border.
5: Compute geometric attributes for the blobs.
6: Weight the blobs by their size and contrast.
7: Sum the weighted blobs to get the index.

In Step 1, a suitable Gaussian low-pass filter is designed to meet the specified lower limit of feature size, $0.5 \mathrm{~mm}$. The cutoff frequency for the filter naturally depends on the image resolution, and size. This step practically removes the dot pattern caused by screening, and softens any isolated defects in print (which are not considered as mottling). In Step 2, the image is thresholded on both sides of the median based on the Weber fraction. From psychometric research, it is known that the human threshold for optical contrast, when expressed as the Weber fraction $d R / R$, tends to be constant over a wide range of reflectances $R$ 8, 14. This suggests that suitable thresholds around the image median can be selected, and the bins for contrast classes can be of equal size. If the mean reflectances of the samples vary considerably, the logarithmic nature (Fechner 


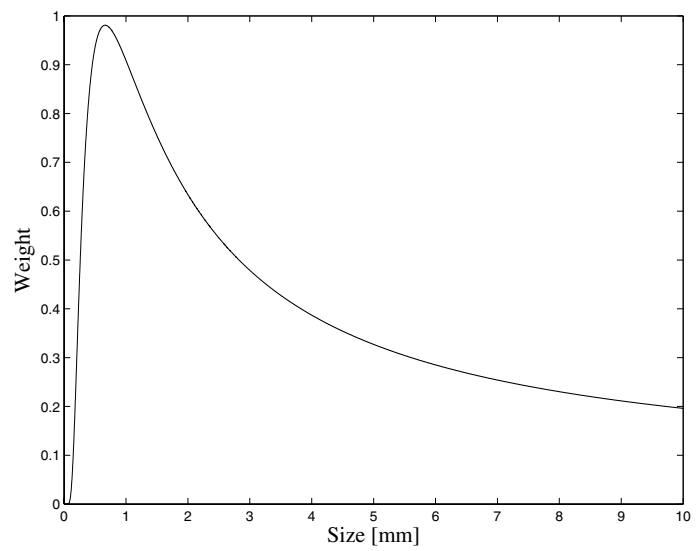

Fig. 1. Weighting of size classes (Mannos CSF)

or some power law) of the sensitivity of the HVS should be considered. In Step 3, morphological opening is used to remove blobs which are too small to be considered as mottling. Also narrow isthmuses between the blobs are cut by the same operator. In Step 4, all blobs in touch with the image border are removed. In Step 5, blob areas are computed as features representing the blobs. In Step 6 , the blobs are divided into 10x10 classes according to their area and contrast. The classes for the area are $0-1 \mathrm{~mm}, 1-2 \mathrm{~mm}, \ldots, 9-10 \mathrm{~mm}$. Blobs larger than $10 \mathrm{~mm}$ are discarded. The classes for contrast are $0-1 \%, 1-2 \%, \ldots, 9-10 \%$. Spots with higher contrast are discarded. A monochrome contrast sensitivity function (CSF) shown in Fig. 1 is used to weight the contrast information of blobs of varying sizes [15. Note that the CSF is derived from perception of sinusoidal patterns, but mottling is a stochastic phenomenon. The number of cycles in a grating visible to a human observer substantially affect the contrast sensitivity 16. However, mottles do not appear as single cycle gratings [13, and thus, we use the one derived using sinusoidal gratings.

Mottling index is computed as a sum of products of size and contrast weights for each blob, i.e.,

$$
M=\sum_{i} W_{a}\left(a_{i}\right) W_{c}\left(c_{i}\right)
$$

where $i$ is the index for a mottle, $a_{i}$ is the area of the $i$ th mottle, $c_{i}$ is the contrast of $i$ th mottle, $W_{a}\left(a_{i}\right)$ is the weight of a size class of the $i$ th mottle, and $W_{c}\left(c_{i}\right)$ is the weight of a contrast class of the $i$ th mottle.

\subsection{Bandpass Method}

This method is based on applying a series of Gaussian band-pass filters to the image in the frequency domain, and computing the coefficient of variation of reflectance $\left(\mathrm{CV}_{R}\right)$ for each image representing a frequency band. Different coef- 


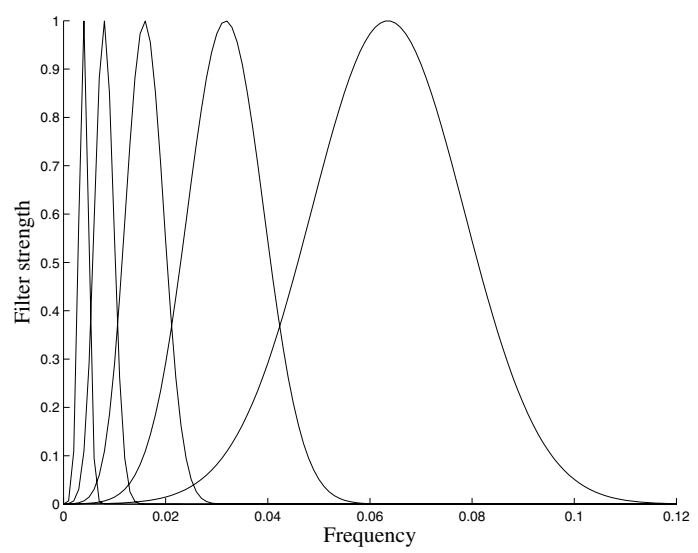

Fig. 2. The filters in $2-\mathrm{D}$ representing the $0.5-1,1-2,2-4,4-8$, and $8-16 \mathrm{~mm}$ spatial bands

ficients represent the difference in reflectance within each band. The coefficients are weighted with the CSF and then summed together as the mottling index 8 . The method is summarized in Algorithm 3 .

\section{Algorithm 3. Bandpass method}

1: Filter the image with a set of bandpass filters separately.

2: Compute coefficients of variation for each band.

3: Weight each coefficient with the CSF.

4: Sum the weighted coefficients to get the index.

In Step 1, the image is filtered in the frequency domain with a series of bandpass filters. Five spatial bands are fixed to an octave series: 0.5-1, 1-2, 2-4, 4-8, and 8-16 mm. The band containing the smallest details has been included when compared to [8]. The Gaussian filters are illustrated in Fig. 2, The DC component is set to 1 so that the mean grey value of the image does not change due to filtering.

In Step 2, the coefficients of variation are computed in the spatial domain for each band. The coefficient of variation is the ratio of standard deviation of reflectance and mean reflectance, i.e.,

$$
\mathrm{CV}_{R}=\frac{\sigma_{R}}{R} .
$$

In Step 3, the coefficients are weighted with a CSF [15] illustrated in Fig. 1] The weights are taken at points representing $0.75,1.5,3,6$, and $10 \mathrm{~mm}$.

\subsection{Visual Assessment}

To compare the results of the implemented methods to human perception, we circulated a set of 20 mottling samples, and asked the human observers to eval- 
uate the perceived mottling. The mean values of these subjective assessments were used as initial reference mottling indices against which the results of all the machine vision methods were compared.

The questionnaire for the assessment consisted of two parts. In the first part, two samples were concurrently compared, and the observer was asked to select the sample which has less mottling. The main function of this part was to present all samples to the observer, and to give some idea of different forms and levels of mottling. These pairwise evaluations could also be used to find possible inconsistencies in the second part. In the second part, each sample was evaluated one at a time, and the observer was asked to rate its level of mottling in a five point Likert scale. There were also two control questions for the observer about the number of times the person had previously evaluated mottling, and the time needed for the test. The primary function of the questionnaire was to evaluate perceived level of mottling of the test set. The secondary, and unwanted, function was to evaluate the person's capability to evaluate mottling and thoroughness of test set evaluation.

The results of the assessments were processed as follows. The people taking the test were divided into two distinct groups based on the control question about the number of times the person has evaluated mottling. The first group consisted of common people who evaluated mottling for the first time and were not experts in the field of print assessment. The second group was formed by experts who evaluated prints as a part of their work. The second control question about the time spent for the test was used to estimate carefulness of the samples evaluation. Selection criteria for outliers were difficult to design. Each observer had his or her own way of selecting the mean value and the use of the scale. However, the mean and standard deviation could be used as elementary criteria to select outliers. If either one differs significantly from the average of all assessments, the assessment was marked as an outlier.

\section{$3 \quad$ Experiments}

We present the results for the $20 \mathrm{~K} 70$ (70\% black) samples. The original samples are approximately $4.5 \mathrm{~cm} \times 4.5 \mathrm{~cm}$ in size. The paper used for printning is 70 $\mathrm{g} / \mathrm{m}^{2}$ LWC (Lightweight Coated) paper and the samples were printed using heatset offset printing process in the KCL layout (KCL heatset layout 01/2003, $60 \mathrm{I} / \mathrm{cm}$, round dot, upper units). The samples were originally scanned with $1200 \mathrm{dpi}$ and 2.2 gamma using flatbed office scanner. The gamma value was not altered before applying the machine vision methods. To reduce computing time, the images were re-sampled to $600 \mathrm{dpi}$.

We inspected mottle sizes ranging from 0.5 to $16 \mathrm{~mm}$ while viewing the sample from a distance of $30 \mathrm{~cm}$ (spatial frequency range $0.03-1$ cycles $/ \mathrm{mm}$ ). Spatially higher- and lower-frequency unevennesses were considered as graininess and banding. Also the inspected contrast of print density was limited to $\pm 10 \%$ of the median grey-value of an image. 


\subsection{Visual Assessment}

These results are based on the 35 human evaluations. The assessments were made in normal office light conditions. However the conditions were not constant, but could vary from one evaluation to another. Evaluators were divided into two groups: experts (12 representatives) and laymen (23 representatives). This division was made based on the professionalism of the person, i.e., the number of mottling evaluations done prior to the suggested one. As it can be seen from Fig. 3(a), there is only a little difference in evaluations between laymen and experts. This is natural since it would be confusing if experts evaluated print quality of samples in which mottling is most visible completely distinctly to endusers. Confidence bounds in Fig. 3(a) present the average results across the whole population \pm standard deviation, and show how similar the mottling indices were among all evaluators.

\subsection{Machine Vision Assessment}

The standard method was implemented in the way it is described in the ISO 13660 standard 2. The implementation of this method is easy and does not

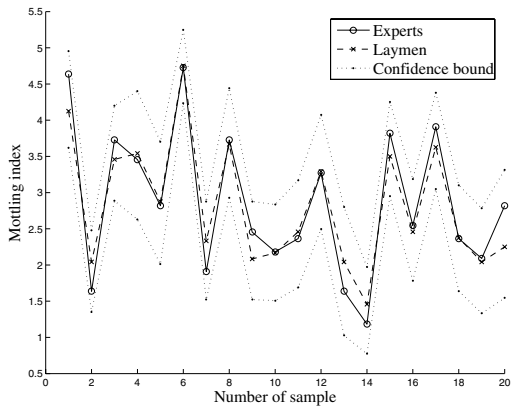

(a)

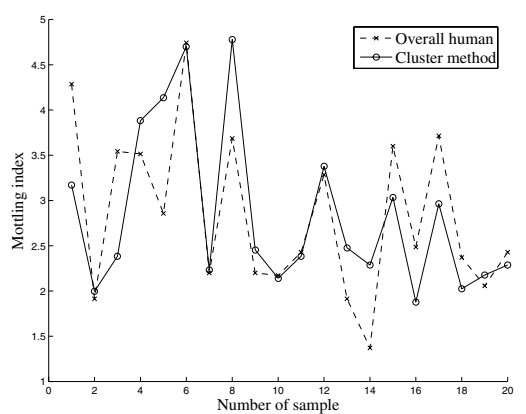

(c)

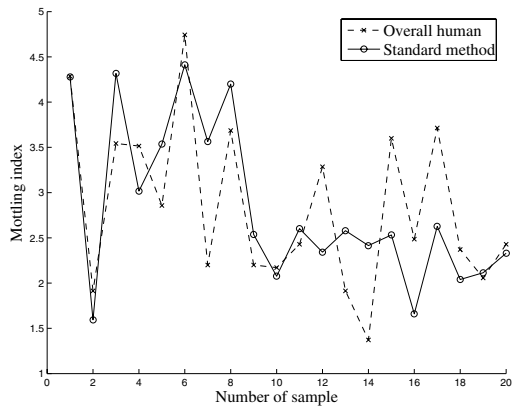

(b)

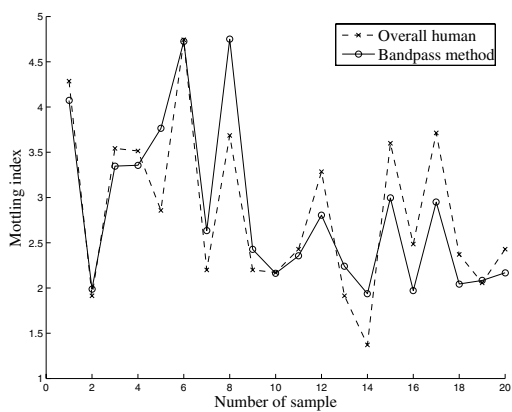

(d)

Fig. 3. Mottling assessments: (a) Human evaluation; (b) Standard method; (c) Cluster method; (d) Bandpass method 
require much programming effort. As it was expected, the results produced by the standard method show low correlation with human evaluation (see Fig. 3(b)) In the standard, the size of the tiles is set to a fixed value which is a known limitation [3]. The cluster method can handle only monochrome images. Another shortcoming is its performance: processing images with a large number of blobs is time consuming. The results of this method can be seen in Fig. 3(c), The bandpass method can also handle only monochrome images. A small number of bands limits the number of spatial classes, and the method becomes similar to a series of low-pass filters used in the early mottling methods. Performance of the method is limited by the resolution of the image and the number of bands. The results of this method can be seen in Fig. $3(\mathrm{~d})$.

All the artificial methods produced mottling indexes in their own scale. Thus, appropriate scaling is needed for the method comparison. We used simple normalization which equalizes mean value and standard deviation of the experimental values across the samples.

\subsection{Results Summary}

In Table1 1 inter-method similarity is presented. Correlation coefficients were used as the similarity measure.

Table 1. Mottling assessment correlations

\begin{tabular}{|l|c|c|c|c|c|c|}
\hline Methods & Overall & Experts & Laymen & Standard & Cluster & Bandpass \\
\hline Overall human & 1.0000 & 0.9848 & 0.9957 & 0.6956 & 0.7330 & 0.8579 \\
Experts & 0.9848 & 1.0000 & 0.9644 & 0.6568 & 0.6717 & 0.8125 \\
Laymen & 0.9957 & 0.9644 & 1.0000 & 0.7078 & 0.7568 & 0.8715 \\
Standard & 0.6956 & 0.6568 & 0.7078 & 1.0000 & 0.6742 & 0.8810 \\
Cluster & 0.7330 & 0.6717 & 0.7568 & 0.6742 & 1.0000 & 0.9070 \\
Bandpass & 0.8579 & 0.8125 & 0.8715 & 0.8810 & 0.9070 & 1.0000 \\
\hline
\end{tabular}

The collected correlation data allow to state that the bandpass method outperforms the other two methods. It can be also noticed that the machine vision methods correlate better among each other than with human evaluation based data. This leads to the conclusion that all artificial methods have a similar nature and the model of human visual system they assume is not accurate. Fig. 3 shows performance graphs for different assessment approaches.

\section{Conclusions}

In the presented work, we performed an initial comparison between human and machine vision evaluation of mottling phenomenon. The results of the human evaluation appear to be highly distributed and, thus, a larger number of assessments is needed both in evaluators and in samples. The high deviation in single 
sample evaluation results leads to the conclusion that a machine vision system modelling an average end-user is necessary. This could bring more precision in delivering printed products of desired quality.

The presented machine vision methods, though having a relatively good correlation with averaged human observation, still need improvement in the sense of modelling of the human visual system. The standard method presented can be considered only as a starting point because this method does not model the HVS at all and also it does not have significant correlation with the human mottling evaluation. The cluster method is based on spatial image processing. This method has some HVS background, but at the same time the approach of "mottle by mottle" processing shows little perspective for improvement. Among the presented methods, the bandpass method shows the best results and it has HVSbased grounds. This method shows potential for improvement and is definitely a candidate for an industrial level machine vision application.

The goals for the future research can be defined as follows:

- Making methods closer to human perception.

- Incorporating mottling evaluation of colour samples.

The general conclusion of our research, is that for the implementation of a machine vision solution to the human perception problem, one needs a suitable HVS model and good statistical characteristics of how the humans perceive the phenomenon.

\section{Acknowledgments}

This work was done as a part of Papvision project funded by European Union, National Technology Agency of Finland (TEKES Projects No. 70049/03 and 70056/04), and Academy of Finland (Project No. 204708).

\section{References}

1. IGT: IGT information leaflet w57: Back trap mottle. WWW:www.igt.nl (2002) [Accessed 2005-02-25]. Available: http://www.igt.nl/igt-site-220105/index-us/wbladen/GST/W57.pdf.

2. ISO: ISO/IEC 13660:2001(e) standard. information technology - office equipment measurement of image quality attributes for hardcopy output - binary monochrome text and graphic images. ISO/IEC (2001)

3. Briggs, J., Forrest, D., Klein, A., Tse, M.K.: Living with ISO-13660: Pleasures and perils. In: IS\&Ts NIP 15: 1999 International Conference on Digital Printing Technologies, IS\&T, Springfield VA (1999) 421-425

4. Wolin, D.: Enhanced mottle measurement. In: PICS 2002: IS\&T's PICS conference, IS\&T (2002) 148-151

5. Armel, D., Wise, J.: An analytic method for quantifying mottle - part 1. Flexo (1998) 70-79

6. Armel, D., Wise, J.: An analytic method for quantifying mottle - part 2. Flexo (1999) 38-43 
7. Streckel, B., Steuernagel, B., Falkenhagen, E., Jung, E.: Objective print quality measurements using a scanner and a digital camera. In: DPP 2003: IS\&T International Conference on Digital Production Printing and Industrial Applications. (2003) 145-147

8. Johansson, P.Å.: Optical Homogeniety of Prints. PhD thesis, Kunglika Tekniska Högskolan, Stockholm (1999)

9. Rosenberger, R.R.: Stochastic frequency distribution analysis as applied to ink jet print mottle measurement. In: IS\&Ts NIP 17: 2001 International Conference on Digital Printing Technologies, IS\&T, Springfield VA (2001) 808-812

10. Barten, P.: Contrast Sensitivity of the Human Eye and its Effects on Image Quality. SPIE (1999)

11. Schade, O.H.: Optical and photoelectric analog of the eye. Journal of the Optical Society of America 46 (1956) 721-739

12. Kang, H.R.: Digital Color Halftoning. SPIE \& IEEE Press (1999)

13. Campbell, F.W., Carpenter, R.H.S., Levinson, J.Z.: Visibility of aperiodic patterns compared with that of sinusoidal gratings. Journal of Physiology (204) 283-298

14. Pratt, W.: Digital Image Processing. A Wiley-Interscience publication (1991)

15. Mannos, J., Sakrison, D.: The effects of a visual fidelity criterion on the encoding of images. IEEE Transactions on Information Theory 20 (1974) 525-536

16. Coltman, J.W., Anderson, A.E.: Noise limitations to resolving power in electronic imaging. In: Proceedings of the Institute of Radio Engineers. (Volume 48.) 858-865 